Marquette University

e-Publications@Marquette

Mechanical Engineering Faculty Research and

Publications

Mechanical Engineering, Department of

$1-1-2013$

Sand Penetration: A Near Nose Investigation of a Sand Penetration Event

\author{
Andrew Van Vooren \\ Marquette University \\ John Borg \\ Marquette University, john.borg@marquette.edu \\ Joshua Felts \\ Naval Surface Warfare Center
}

Published version. Procedia Engineering, Vol. 58 (2013): 601-607. DOI. (C) 2013 Elsevier. Used with permission. 


\title{
Sand Penetration: A Near Nose Investigation of a Sand Penetration Event
}

\author{
Andrew Van Vooren ${ }^{\mathrm{a}}$, John Borg ${ }^{\mathrm{a} *}$, Harold Sandusky ${ }^{\mathrm{b}}$ and Joshua Felts ${ }^{\mathrm{b}}$ \\ ${ }^{a}$ Marquette University, 1515 W. Wisconsin Ave., Milwankee 53233, WI USA \\ ${ }^{b}$ Naval Surface Warfare Center, Indian Head, MD, USA
}

\begin{abstract}
This paper presents experimental and computational results of a long-rod penetrating dry granular sand at velocities near $100 \mathrm{~m} / \mathrm{s}$. The objective of this work is to develop a fundamental understanding of the formation and transmission of dynamic force chains, and the motion and fracture of the individual sand grains as the projectile passes. This is accomplished by launching a projectile along a view window, backed by sand, in order to directly view and photograph the projectile/sand interactions. Within the sand system, a two-wave structure was observed, composed of a compaction wave (bow shock) that detaches from the dart and moves through the sand at a wave speed near $100 \mathrm{~m} / \mathrm{s}$ and a damage wave, which remains near the leading edge of the dart. The compaction wave removes porosity and the damage wave fractures grains in the region near the projectile nose. Grain fracture is not observed at dart speeds below $35 \mathrm{~m} / \mathrm{s}$. In addition the axial strain to failure of individual sand grains was measured in a quasi-static configuration. These results were used in conjunction with a simple analytic force balance model to predict the depth of penetration. The analytic results compare favourably with experiments until the dart slows below $35 \mathrm{~m} / \mathrm{s}$.
\end{abstract}

(C) 2013 The Authors. Published by Elsevier Ltd. Open access under CC BY-NC-ND license.

Selection and peer-review under responsibility of the Hypervelocity Impact Society

Keywords: Sand; heterogenous material; penetration; granular material.

\section{Introduction}

The penetration dynamics of a projectile launched into a heterogeneous target have long been of interest and remain an active research area. Experimental investigations have demonstrated that the penetration behavior of a projectile can vary against differing heterogeneous materials such as soil [1-4], sand [5-19], concrete [20-23], as well as laminated and/or composite materials. Soil, specifically loose dry sand, has demonstrated a wide range of behaviors when subjected to shock and impact loading. Grain fracture, pore collapse and the formation of force chains are some of the important phenomena which govern the sand behavior at the small scale, complicate the dynamic target-projectile interactions moving up in scale, and are suspected to contribute to differing degrees of penetration performance at the largest scales. The objective of this work is to explore the formation of force chains, and to observe and quantify the fracture of individual sand grains when subjected to a penetration event. This is accomplished with a novel experimental technique that uses an optically accessible target tank in order to directly observe the projectile-target interactions.

Impact and penetration of loose dry materials, such as sand, have been modeled utilizing a variety of empirical, analytic and numeric techniques. Simple analytic hydrodynamic approximations have successfully been used to predict the bulk behavior of a wide variety of projectile-target configurations, including materials with porosity [24-25]. Often the performance of a penetration event is based on bulk postmortem metrics such as depth of penetration, projectile erosion,

* Corresponding author.E-mail address: john.borg@mu.edu. 
shot-line deviations, crater formation and excavation. Often the depth of penetration is compared to the hydrodynamic limit, defined as the ratio of penetration depth to projectile length and is proportional to the square root of the ratio of the projectile and target densities, $\mathrm{P} / \mathrm{L} \sim \sqrt{\rho_{p} / \rho_{00}},[26,27]$. For the projectile and sand targets investigated here, the measured $\mathrm{P} / \mathrm{L}$ ratio is larger than 5 , whereas the square root of the density ratio is near 2 . Thus this estimate does not accurately characterize the depth of penetration. Charters and Summers suggested estimates of penetration can be made by equating the kinetic energy of the projectile to the target resistances times an assumed excavated volume [28]. The volume estimated can either be a hemisphere, as originally assumed by Charters and Summers, or a cone, where the diameter of the cone is equal to the penetration depth; both are presented in Equation 1.

$$
P=\left(\frac{81}{4 \pi} \frac{\rho_{p}}{\rho_{t}} \frac{K E}{S t}\right)_{\text {Hemi }}^{1 / 3}=\left(\frac{162}{\pi} \frac{\rho_{p}}{\rho_{t}} \frac{K E}{S t}\right)_{\text {Cone }}^{1 / 3}
$$

The target resistance, $S t$, is assumed to be the flow stress and therefore an expression for the depth of penetration can be obtained. In addition, we propose a simple analytic solution based on a simple force balance and the resistance due to individual grain fracture. Reviews of empirical and approximate methods can be found in Zukas, et. al. [29] and Orphal [30].

\section{Experimental setup}

Figure 1a presents a photograph of the dynamic test setup, showing the orientation of the horizontally mounted air driven gas gun, the target box and the high-speed camera. Figure $1 \mathrm{~b}$ presents a photograph of the gun barrel, stripper box and target tank. Experiments were conducted such that the $4.5 \mathrm{~mm}$ diameter air gun launches a projectile into a $35 \mathrm{~cm}$ (shot direction $) \times 25 \mathrm{~cm} \times 18 \mathrm{~cm}$ tank filled with Ottawa sand $(0.45-0.55 \mathrm{~mm}$ average grain diameter, average bulk density: 1.56 $\left.\mathrm{g} / \mathrm{cm}^{3}\right)$. The sand used in these experiments consists of $99 \%$ pure quartz $\left(2.65 \mathrm{~g} / \mathrm{cm}^{3}\right)$, have rounded grains and are rather light transparent; thus they appear clear. The bulk porosity of the sand is $39 \%$.

The objective was to gain visual access to the semi-infinite target and produce two-dimensional planar photographs of the penetration event. Thus the target tank was constructed from optically accessible $1.0 \mathrm{~cm}$ thick polycarbonate (i.e. bullet proof glass) and the gun barrel was aligned to the tank such that the projectile remained in near contact with the polycarbonate view window for the first portion of the penetration event. A similar technique was used in the exploration of low speed impacts and impact crater formation due to small charge explosives [31,32]. The aluminum darts used in these experiments were $10.16 \mathrm{~cm}$ long and $0.635 \mathrm{~cm}$ diameter with a flat nose and were launched with a full-bore $5 \mathrm{~cm}$ long nylon sabot. After exiting the barrel, the dart free flies through the stripper box which acts as a blast chamber. The dart enters a tapered section that guides it into the target box and along the view window. The dart penetrates a make-switch, which triggers the camera and oscilloscopes, just before entering the target box. The sabot is separated from dart in the stripper box by impacting a stripper plate once the dart is nearly $1 \mathrm{~cm}$ into the sand. Two quartz PCB Piezotronics $208 \mathrm{C} 01$ pressure gauges were buried in the sand to measure the passing stress wave.

The penetration event was photographed using a Photron RS and/or a Cordin 550 camera. The Photron camera was used to visualize nearly the entire sand box field of view (FOV) of $26 \mathrm{~cm} \times 22 \mathrm{~cm}$ with frame rates near 12,000 frames per second and a resolution of $512 \times 432$. The Cordin camera was configured to take 64 images at a resolution of $1024 \times 1024$ pixels with a FOV of $2.7 \mathrm{~cm} \times 2.7 \mathrm{~cm}$ at an acquisition rate near 120,000 frames per second.

An aspect of these experiments that becomes important when viewing the results is the orientation of the camera and lighting. The cameras were placed approximately $36 \mathrm{~cm}$ from, and right angles to, the target window. Lighting was accomplished with two halogen lights placed approximately $72 \mathrm{~cm}$ from the target view window, behind the camera and slightly off angle in order to avoid bright spots in the camera FOV. Thus all the light collected by the camera was reflected from the sand grains. This is an important aspect of the photographic record where the light measured by the camera increases due to an increase in reflected light from fractured sand grains. This will be discussed in greater detail below.

\section{Results}

\subsection{Initial experimental results}

In the initial experiments a two-wave structure was observed propagating through the sand that was composed of a compaction wave (or bow shock) and a damage wave. The compaction wave, which detaches from the projectile and propagates through the sand at a speed near $100 \mathrm{~m} / \mathrm{s}$, removes porosity through the translation and rearrangement of grains 
and results in the formation of force chains. The damage wave fractures grains in the region near the nose of the projectile. Evidence of this two-wave structure can be seen in Fig. 2, where the dart travels from right to left. The compaction wave is best identified by viewing a series of images (i.e. watching a movie) thus it has been highlighted here for clarity. The dart appears as a blur in the center-right of the Fig. $2 \mathrm{~b}$ and $2 \mathrm{c}$. Given the relatively low framing rate of these imagines the compaction wave and force chains appear as streaks. The damage wave appears as a brighter conical region near the nose of the projectile. Later, experiments were done to confirm that the fracture cone around the dart resulted from an increase in reflectivity from the increased surface area of the fractured grains. This will be discussed in greater detail below. From these initial experiments it was determined that the fracture cone was visible at launch velocities above $35 \mathrm{~m} / \mathrm{s}$, which means that at velocities above $35 \mathrm{~m} / \mathrm{s}$ sand grain fracture is an important mechanism in the penetration event.

In order to further investigate the fracture cone region, high magnification and high framing rate images were obtained with a 1 megapixel Cordin 550 camera. This camera was used to obtain images at 175,000 frames per second. With this higher magnification individual grains can be tracked and the formation of force chains and fracture can be observed. Figure 3 presents a series of photographs in which the dart is traveling from right to left with an initial velocity near 100 $\mathrm{m} / \mathrm{s}$. These images illustrate individual grains being pushed together and rearranging, until coming into closer contact with other grains and forming the force chains. Grains within these force chains increase in reflected light and subsequently fracture. An individual grain involved in this process is highlighted in Fig. 3a with a square and a magnified view of this grain as it forms a force chain and fractures is presented in the right column. This grain is initially ahead of the fracture wave in Fig 3a. It becomes involved in a 3-to-5 grain force chain in Fig. 3b and there appears within the grain a brighter spot, i.e. an increase in reflectance. We believe this to be a result of grain damage, i.e. an increase in fracture surfaces within the grain, which results in greater reflectivity. This will be discussed in more detail in the following section. Finally the grain catastrophically fractures and shatters into many smaller shards in Fig. 3c. There are multiple bright spots that emanate from the fractured shards. Note the total translation of the grain is quite small. It has fractured near the same location from which it started. Also note that to the right of the fractured grain there appears a rather diffuse, bright cloud, where it is difficult to distinguish individual grains. This is a collection of fracture grains, the fracture wave, and is the source of the "white trail" left behind by sand penetration which has been observed by others $[6,19]$.

a)

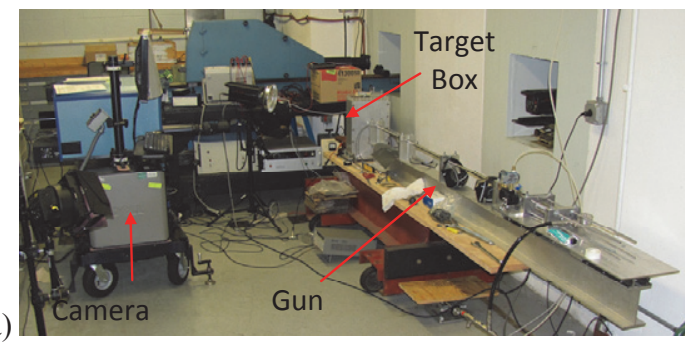

b)

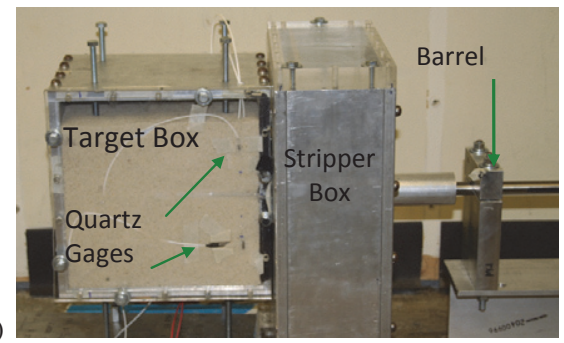

Fig. 1. Experiment setup of the a) the gun and camera setup and b) the target box.
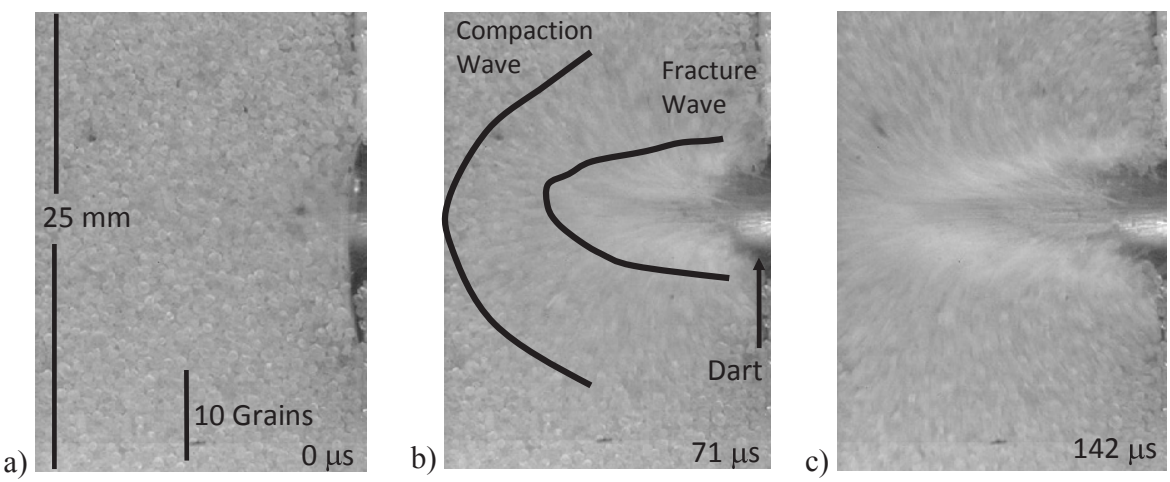

Fig. 2. Three frames $71 \mu \mathrm{s}$ apart, showing the progression of the two-wave structure: compaction and fracture. Dart is moving right to left $100 \mathrm{~m} / \mathrm{s}$ 


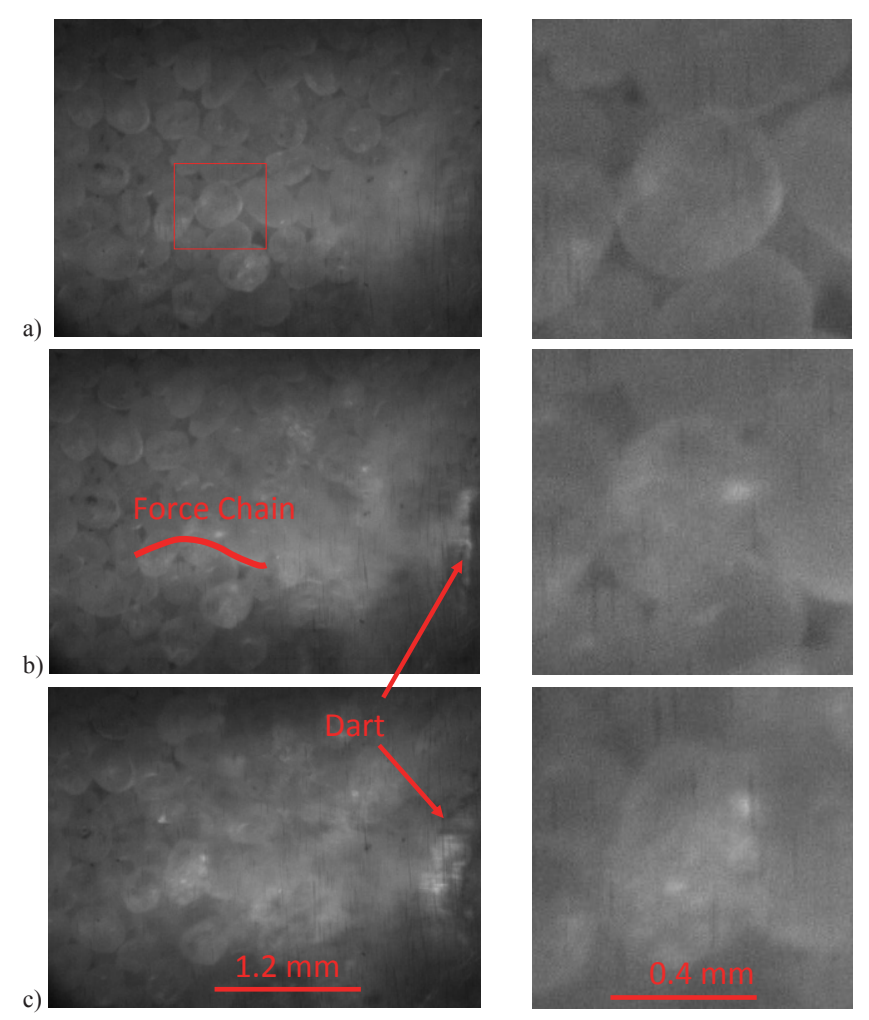

Fig. 3. Frames showing the progression from free grain to stress bridge formation to grain fracture. The right column presents an individual grain, highlighted in red in frame a) of the left column, undergoing a dynamic fracture event. The time between frames a) to c) is approximately $80 \mu s$.

Based on viewing multiple video records, it appears that for impact velocities near $100 \mathrm{~m} / \mathrm{s}$ the force chains are on average 5-10 grains long. At any given frame of the video, approximately $75 \%$ of the grains will be involved within a force chain. Thus one fourth of the grains in the FOV are free floating and not involved in a force chain. It is also important to note that there is very little grain rotation in front of the dart, grains are generally pushed in the direction in which the projectile is traveling. Finally, not all grains are involved in a force chain fracture. Only one grain needs to fracture to release the stress within the chain; the remaining grains are then free to be taken up by new force chains. With these experiments, the FOV is such that only grains directly in front of the projectile are viewed. In future testing we plan to move the FOV to view the shear regions to the side of the dart.

\subsection{Single grain experiments}

This set of experiments was composed of two parts; the first was focused on observing the phenomenon wherein grains of sand would increase in reflectivity when experiencing an external force, and determining whether or not this increase was reversible once the force was removed and the second part of this experiment was aimed at gathering data on the stress/strain relationship for single grains of sand.

In the first experiment, a single grain of sand was placed in between two metal plates, and a force (i.e. pressure) was quasi-statically increased until the grain began to fracture and then eventually was completely crushed. This process was filmed with the Photron high speed camera, and a $1 \mathrm{~kW}$ Halogen light. As mentioned above, the lighting was also placed behind the camera so that all the light collected is reflected light. This process of fracturing and crushing a grain was repeated 25 times. It was observed that the grains form crack faces which propagate throughout the grain, and this face is responsible for the increased reflectivity. Figure 4 shows the frame before a) and the frame after b) a crack face has formed ( 1 thousandth of a second apart); note the increase in reflectivity. Nearly identical phenomena has been observed in more controlled tests of pure quartz samples in a split Hopkinson bar configuration [33]. 
Although the grain has increased in reflectance it has not visibly failed, it has only been damaged. The grain remains intact until further strain causes it to fail. In addition we appreciate that some fracture surfaces must form in an orientation such that light does not reflect back to the camera. Given the grain morphology, the planarity of the fracture surface and the angle of the light to the camera, it is unclear what percentage of the fracture surfaces can be detected using this method. If we correlate an increase in reflected light to damage and fracture, it is likely that more damage and fracture has occurred than is evident by an increase in reflectivity. However, on average, a strong correlation can be made.

In order to make certain that this phenomenon was not the result of a force or strain dependent luminescence this experiment was repeated in low light conditions. When this was done the camera did not measure any light; thus it was concluded that the increase in measured light is due to reflectance and not luminescence. Although it cannot be conclusively determined that the grain does not luminesce, such low levels of illumination (if present) are not detectable by our cameras.

When a video of a grain fracture event is viewed, the grain progresses from intact, then multiple fracture events occur, and eventually the grain fails catastrophically, Fig. 5. Typically shards of the grain spall before the grain catastrophically fails. For each different fracture a different amount of force (i.e. work) would be required to cause additional fracture surfaces to be created. This shaped the way in which the second experiment was carried out.

In the second group of quasi-static experiments, a single grain was crushed using a force transducer on the top platen and a flat metal fixture on the bottom. A video of the grain being crushed was taken while collecting data from the force transducer. The camera and force transducer were synchronized. After the experiment was completed the video results were analyzed. The initial height of the grain was measured in pixels and was used as the base height since no force had yet been applied to the grain. This frame was also used to calculate the approximate cross-sectional area by assuming that the width and depth were equal. Then the frame before the first time the grain fractured was found, and once again the height of the grain in pixels was measured. The time that this frame occurred was used to find the corresponding force from the oscilloscope data. This was then repeated for the last fracture before the grain was completely crushed. These two data points were recorded as the first and second fractures respectively. This was repeated twenty times, and the results are presented in Fig. 6. These results are not intended to be a true stress-strain relationship. Given the morphology and composition of individual grains, the pre-damage and loading direction, these results represent a statistical envelope of axial loads in which one might expect grains to fracture. These results may be useful in giving an approximate average stress/strain that a single grain will undergo before catastrophically fracturing, which can be used in modelling the dart penetration experiment. In addition, the individual stress strain result obtained from controlled Hopkinson bar experiments on $\alpha$-quartz is presented in Fig. 6 [33]. This result gives credence to the quasi-static results obtained here.
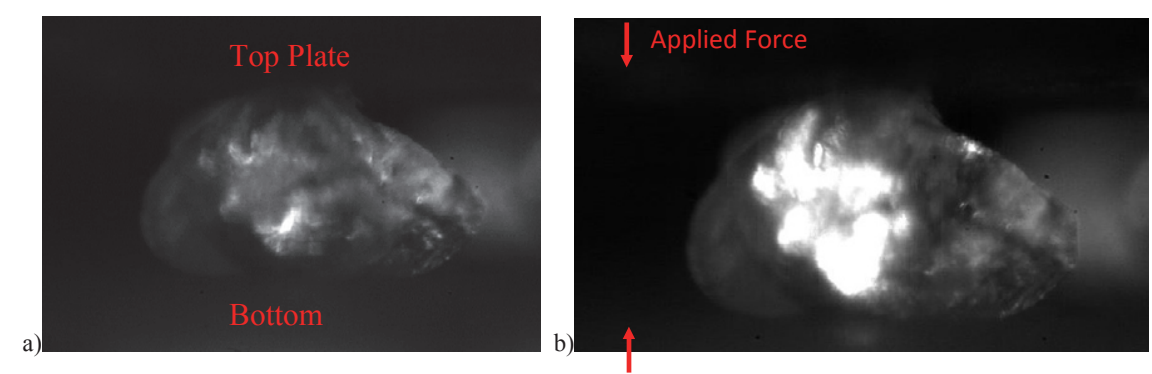

Fig. 4. Frames showing the progression of a grain from a) intact to b) fractured with increased reflectivity. The height of the grain is $0.486 \mathrm{~mm}$.
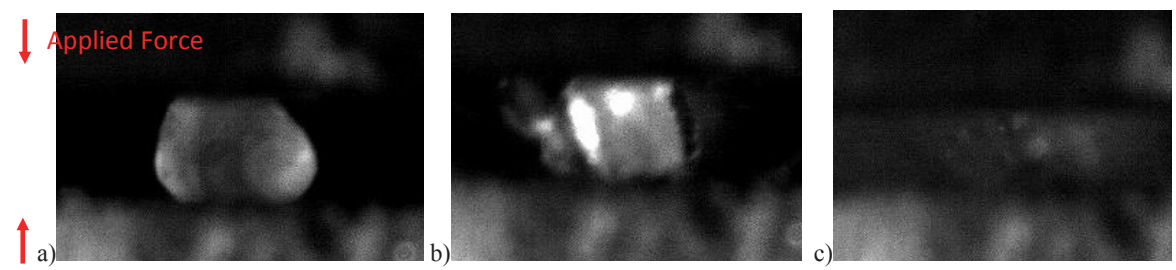

Fig. 5. Frames showing the progression of a grain from (a) intact to (b) fractured with fragments flying off to (c) catastrophically fractured. 


\subsection{Numerical Calculations}

A simple model consisting of a force balance can be constructed by considering only axial stress, $Y$, acting on the nose of the dart,

$$
\begin{gathered}
d^{2} x / d t^{2}=\beta Y A / m \\
x(t)=\mathrm{v}_{0} t+\beta Y A t^{2} / 2 m
\end{gathered}
$$

where $m$ and $A$ is the mass and frontal area of the dart respectively. The stress acting on the dart is taken to represent only grain fracture, as presented in Fig. 6, where the average axial fracture stress is near 0.5 GPa. This equation can be integrated to give Equation (3), where $v_{0}$ is the initial velocity of the dart. Since the dart surface area is in contact with individual grains and since not all grains fracture, included is a factor $\beta$, which is less than one and which reduces the total force acting on the nose. Beta was selected to be the solid volume fraction of the sand, 0.55 , times the fraction of grains observed to fracture, 0.1 , which results in a $\beta$ factor of 0.055 . Figure 7 presents this analytic solution, Equations (2) and (3), along with Equation (1), the Charters and Summers relation, as compared to the experimental data. The simple analytic solution predicts the early time dart penetration where grain fracture dominates the forces acting on the nose of the dart. As the dart velocity drops, and grain fracture is no longer observed, the analytic solution under predicts the dart penetration depth. Thus we conclude that other, lower magnitude forces, such as excavation and friction dominate the flow. The Charters and Summers relation predicts a total penetration depth of $2 \mathrm{~cm}$, which is quite low compared to our data.

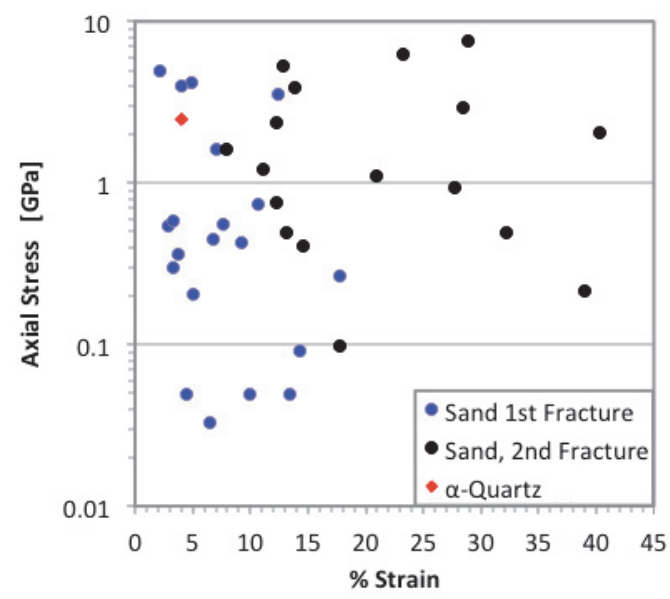

Fig. 6. Plot of "stress vs strain" for a single grain of sand and a Hopkinson bar data point for pure quartz [33].
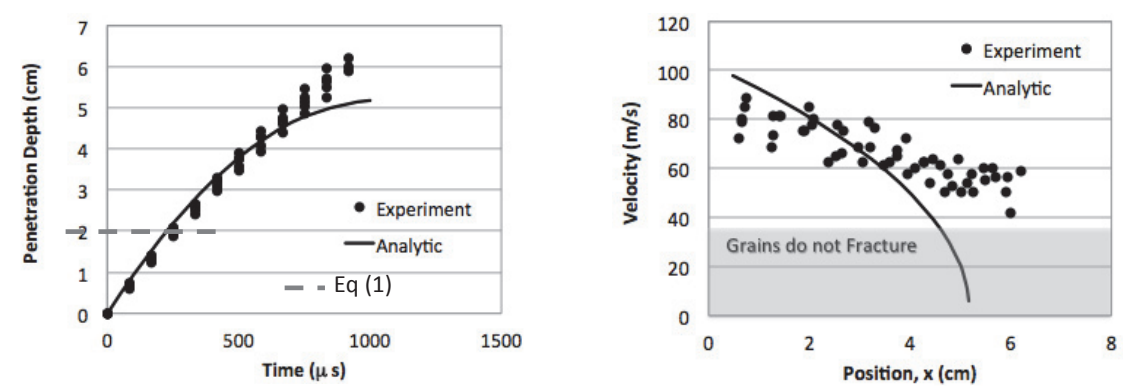

Fig. 7. Comparison of experimental and analytic penetration behavior. Below a threshold velocity near $35 \mathrm{~m} / \mathrm{s}$ grains do not fracture. 


\section{Conclusions.}

This work demonstrates a fundamental mechanism in the penetration of granular materials, namely the formation of force chains and dynamic fracture. Although this process has been observed in static experiments and demonstrated numerically, these are the first experiments (that we are aware of) that dynamically capture this phenomena experimentally. Grain fracture was not observed at velocities below $35 \mathrm{~m} / \mathrm{s}$ but instead grains are displaced as the dart progresses through the sand. A quasi-static experiment was used to estimate the fracture strength of individual sand grains, the results of which were used to construct an analytic solution for the penetration event. This analytic solution under predicts the depth of penetration when grain fracture is no longer observed.

\section{Acknowledgements}

This work was funded by the Defense Threat Reduction Agency (DTRA) under contract HDTRA1-09-0045. We would like to thank Drs. Richard Lewis and Su Peiris for serving as program managers.

\section{References}

[1] Byers, RK, Chabai, AJ and Walsh RT. "Predictions of projectile penetration phenomena and comparison with experiments in a soil medium" Sandia National Laboratories Report 75-0174, 1975.

[2] Bernard RS and Creighton DC. "Projectile penetration in Soil and Rock: Analysis for Non-Normal Impact," US Army Engineer Waterways Experiment Station, Technical Report SL-79-15, 1979.

[3] Richmond, PW. "Influence of nose shape and L/D ratio on projectile penetration in frozen soil," US Army Cold Regions Research and Engineering Laboratory, Special Report 80-17, 1980.

[4] Forrestal, MJ, and Luk VK. "Penetration into soil targets," Int. J. Impact Eng., 12, 427-444, 1992.

[5] Allen, WA, Mayfield, EB and Morrison HL. "Dynamics of a projectile penetrating sand," J. Appl. Physics 28(3), 370-376 (1957).

[6] Allen, WA, Mayfield, EB and Morrison HL. "Dynamics of a projectile penetrating sand. Part II," J. Appl. Physics 28(11), 1331-1335 (1957).

[7] Wang WL "Experimental study of projectile penetration in Ottawa sand at low velocities", J. Spacecraft Rockets, 6 (4) 497-498 (1969).

[8] Savvateev, AF, Budin, AV, Kolikov, VA and Rutberg PG. "High-speed penetration into sand," Int. J. Impact Eng., 26 (1), 675-681 (2001).

[9] Houa, M., Penga, Z., Liua, R., Wua, Y., Tiana, Y. Lua, K. and Chan, C.K. Projectile impact and penetration in loose granular bed. Science and Technology of Advanced Materials 6, 855-859 (2005)

[10] Bless, S.J., D., Berry, T., Pedersen, B. and Lawhorn, W., Sand Penetration By High-Speed Projectiles. Shock Proceedings from the Shock Compression of Condensed Matter-2009, Nashville, TN (2009).

[11] Boguslavskii, Y., Drabkin, S. and Salman, A., Analysis of vertical projectile penetration in granular soils. J. Phys. D: Appl. Phys. 29 905-916 (1996).

[12] Collins, A.L., Addiss, J.W., Walley, S.M. Promratana, K., Bobaru, F., Proud, W.G. and Williamson, D.M. The effect of rod nose shape on the internal flow fields during the ballistic penetration of sand. Int. J. Impact Eng. 38, 951-963 (2011)

[13] Collins, J.A. and Sierakowski, R.L., Studies On The Penetration Of Eglin Sand. AFRL Report AFATL-TR-76-122 (1976).

[14] Forrestal, M.J., Longcope, D.B., and Lee, L.M., Analytical and Experimental Studies on Penetration into Geological Targets, from the proceeding of The Interaction of Non-Nuclear Munitions with Structures, US Air Force Academy, Colorado, Report AD-A132-115, pg 23-26, May, (1983).

[15] Savvateevetal, A.E., High-Speed Penetration Into Sand Int. J. Impact Eng. 26, 675-681 (2001)

[16] Stone, G.W., Projectile Penetration into Representative Targets Sandia Report 1490 UC-704 October(1994)

[17] Taylor, T., Fragaszy, R.J. and Carlton, L.H., Members, ASCE., Projectile Penetration In Granular Soils. J. Geotech Eng-ASCE., 117, 4, (1991).

[18] Wang, W.L., Experimental Study of Projectile Penetration in Ottawa Sand at Low Velocities. Engineering Notes, pg. 497, April (1969)

[19] Watanabe, K., Tanaka, K., Iwane, K., Fukuma, S., Takayama, K., and Kobayashi, H. Sand Behavior Induced by High-Speed Penetration of Projectile. AFOSR Report, AOARD-094011, Tokoyo, Japan, Feb (2011)

[20] Yankelevsky, DZ. "Local response of concrete slabs to low velocity missile impact.” Int. J. Impact Eng., 19, 4, 331-343 (1997).

[21] Corbett, GG, Reid, SR and Johnson, W. "Impact loading of plates and shells by free-flying projectiles: A review." Int. J. Impact Eng., 18, 141-230 (1996).

[22] Forrestal MJ, Altman, BS, Cargille JD, and Hanchack SJ. "An empirical equation for penetration depth of ogive-nose projectiles into concrete targets." Int. J. Impact Eng. 15(4) 395-405 (1994).

[23] Gran, JK and Frew DJ. "In-target radial stress measurements from penetration experiments into concrete by ogive-nose steel projectiles", Int. J. Impact Eng. 19(8) 715-726, (1997).

[24] Tate, A. "A theory for the deceleration of long rods after impact," J. Mech. Phys. Solids, 15 387-399 (1967).

[25] Grove, B. "Theoretical considerations on the penetration of powdered metal jets" Int. J. Impact Eng., 33, 316-325 (2006).

[26] Evans W. M. and Pack, D.C. Penetration by High-Velocity ('Munroe') Jets: II Proc. Phys. Soc. B 64303 (1951).

[27] Tate, A. Long Rod Penetration Models Part II. Extensions To The Hydrodynamic Theory Of Penetration. Int. J. Mech. Sci. 28 (9), 599-612 (1986).

[28] Charters, A.C. and Summers, J.L. Comments on the Phenomena of High-Speed Impact. NOLR1238, U.S. Naval Ordance Lab., White Oak, 200-221 (1959).

[29] Zukas, JA, Nicholas, T, Swift, HF, Greszczuk LB and Curran, DR. Impact Dynamics, Kriger Publishing Company (1992).

[30] Orphal. DL. "Explosions and Impacts" Int. J. Impact Eng., 33, 496-545 (2006).

[31] Houa, M., Penga, Z. Liua, R. Wua,Y Tiana, Y., Lua, K and Chanb, C.K., Projectile impact and penetration in loose granular bed. Science and Technology of Advanced Materials 6 855-859 (2005)

[32] Piekutowski, A., Formation of bowl-shaped craters. Proc. Lunar Planet. Sci. Conf. 11th, 2129-2144 (1980).

[33] Kimberley, Ramesh and Barnouin, Visualization of the failure of quartz under quasi-static and dynamic compression, J. GEOPHY R., 2010 